\title{
The Importance Of Gender And Facial Information Science On Information Systems Design
}

Ralph Reilly, University of Hartford, USA

Andrew Nyaboga, William Paterson University, USA

Carl Guynes, University of North Texas, USA

\begin{abstract}
Facial Information Science is becoming a discipline in its own right, attracting not only computer scientists, but graphic animators and psychologists, all of whom require knowledge to understand how people make and interpret facial expressions. (Zeng, 2009). Computer advancements enhance the ability of researchers to study facial expression. Digitized computer-displayed faces can now be used in studies. Current advancements are facilitating not only the researcher's ability to accurately display information, but recording the subject's reaction automatically. With increasing interest in Artificial Intelligence and man-machine communications, what importance does the gender of the user play in the design of today's multi-million dollar applications? Does research suggest that men and women respond to the "gender" of computer displayed images differently? Can this knowledge be used effectively to design applications specifically for use by men or women? This research is an attempt to understand these questions while studying whether automatic, or pre-attentive, processing plays a part in the identification of the facial expressions.
\end{abstract}

Keywords: Facial Information Science, Gender Difference, Systems Design, Visual Field Interaction, nonverbal communication

\section{INTRODUCTION}

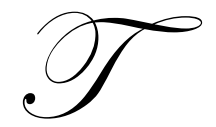

he purpose of the research presented in this dissertation has three goals: first, an attempt to determine if gender is significant in reaction to facial affect; second, to address the question of whether a distractor plays a role in the processing of facial expression; and third, an attempt to understand the information systems implications involved in such a study. Early researchers suspected that the ability of an individual to express and interpret nonverbal messages was an important factor in his ability to communicate with others. However, early studies of nonverbal communication were unsuccessful because they employed static photographs of posed emotional expressions. In later studies, emotional responses were produced through the presentation of emotionally loaded stimuli.

In studies by Stanners, Byrd and Gabriel (1985), there was evidence to show that females identified pleasant female facial expressions more quickly than any other target/subject combination. Zajonc's studies (1980) argue that a decision is based on an early affective (like-dislike) reaction to the image rather than a feature discrimination process. The researchers found that female pairs were more effective in the transmission and reception of nonverbal emotional cues than were the male pairs. It was uncertain whether this was due to a more overt and "readable" nonverbal signal from the female senders than from the male senders or to a heightened sensitivity to nonverbal cues by the female observers. Indeed, both factors could be involved. Both male and female pairs showed negative relationships between the physiological responding of the sender subject and the accuracy of communication. 


\section{FACIAL PROCESSING}

Facial processing engages several separately localized cognitive mechanisms. According to Hillger and Koenig (1991), there is evidence that separate left- and right-hemisphere mechanisms are involved in face processing. Some of them appear to be general-purpose visual mechanisms that are more efficient in the lefthemisphere, whereas others appear to be specialized (though not necessarily face-specific) mechanisms that are more efficient in the right hemisphere. The left-hemisphere seemed able to detect differences in single facial features (parsing). These mechanisms are probably engaged simultaneously when a stimulus is presented; the mechanism that is most appropriate for the judgment wins - i.e. produces a response faster and more accurately (Hillger and Koenig, 1991).

Prior studies indicate that the female has a superior ability to recognize facial expressions of emotion(Buck, Miller, Caul, 1974; Buck, 1979; Hall, 1984).

In examples conducted using photographs of male and female faces displaying anger, fear, sadness and disgust. These studies seem to further indicate that females are more accurate at revealing emotion than the male. The single exception being anger, which was more readily recognized when expressed by men than by women. It might also be expected that females are able to identify expressions more rapidly than males (Stanners, Byrd, and Gabriel, 1985). The implication of the Stanners, Byrd and Gabriel study is that females (relative to males) have some specialized capacity for processing female faces.

Davitz (1964) and Tagiuri (1969) hypothesized that the "gender effect" (a term they coined to explain the gender encoding/decoding capabilities of the male versus female) varied appreciably with the gender of the sender (or target, as referenced in this study). However, in Hall's later study(1984), it was found that the gender effect did not vary with the gender of the sender (or target). Hall notes that females exceed males at all ages in their recognition of emotions and that women are also better senders (facial transmitters) of emotion than men.

\section{GENDER AND PERSONALITY}

Buck and Miller (1974) explored possible relationships between gender and personality variables, and the negative correlation between communication accuracy and physiological responding, as previously explained. The study demonstrated significant communication of emotion via facial expression using both the categorization and pleasantness measures of communication accuracy. The results indicated that the superior communication found among female pairs was due to the greater facial responsiveness of the female senders. Female senders demonstrated more accurate communication than male senders, but female observers were not found to be reliably more accurate than male observers. Females were also rated as being more facially expressive than males. Males had a tendency to be internalizers while females tended to be externalizers. This was related to cultural influences, since in our society males are generally discouraged more from overly expressing most emotions than are females.

The idea that women respond to emotional stimuli by an activation of emotion processing systems, and men respond with a reactive inhibition is suggested in the research by Burton and Levy (1989). Their data showed that reaction times to negative emotions are faster overall than to positive emotions. Another study suggests that women are more emotionally expressive than men (Strouse, 1974), and that response times to negative emotions take longer in the right visual field than in the left visual field, whereas response times to positive emotions are longer in the left visual field. The effect was found to be significant among females but not among males. They suggested that the intensity of induced emotion may be greater for females than males due to the elaborative responses in females and the reactive, inhibitory responses in men.

\section{VISUAL FIELD INTERACTION}

In a face-to-face task, comparisons of a centrally presented and a laterally presented emotional face were required. The Emotional Valence (positive or negative response) by Visual Field Interaction (left visual field or right visual field) testing found that reaction times in the left visual field were faster for negative emotions presented in 
the left visual field. In addition, reaction times to positive emotions were faster when presented in the right visual field. An interaction of gender, task, and emotional valence was found. There were opposite effects for the two genders (Burton \& Levy). The reason for the opposite responses is that there is generally greater right hemisphere superiority for women for expressing facial emotion. The right cerebral hemisphere is specialized for interpretation and communication of emotion (Landis, Assol, \& Perret, 1979; Safer and Levanthal, 1977). If this is true, then it follows that females should respond more quickly and accurately than males in an emotion identification task. In the studies, evidence was found that indicated that left visual field presentations resulted in superior overall response times, irrespective of the affective valence of the stimulus of the gender of the subject. They also found evidence of overall better recognition for the positive emotions as compared to the negative emotions across visual half-fields. Additionally, men showed superior emotional identification with the left visual field while women did not. Their study indicated that men were faster than women in attempts to tap right hemisphere identification of facial expressions. On the surface, then, it would appear that the results support the contention of a more different lateralization among women for the processing of affective information. (Harrison et al, 1990).

\section{EMOTION}

In a study by Fasel et al, evidence was found that male subjects, as compared to female subjects, were selectively less sensitive to sad emotion in female faces. Female subjects were more sensitive overall to emotional expression in male faces than in female faces. In their study men and women differed in performance depending on the gender of the facial stimuli (Fasel 2003).

Further, they found that women are generally more sensitive to happy than sad faces and are more sensitive to male than female faces, whereas men are equally sensitive to happy and sad faces in men but substantially less sensitive to sadness in women's faces. Analysis of their results lead them to conclude that controlling for the gender of the facial image displayed moderated the male advantage. They suggested further study in the relationship of gender between the displayed image and the subject.

This research poses the question of whether the subjects' gender effect is independent of the gender of the stimulus face. In other words, does the gender of the displayed image play a role in the response of the subject? Bourne (2005) discovered that male faces were overall better recognized than female faces, and particularly the negative male face. Male negative faces also required longer VRTS (visual response time - the time required from the initial display of the facial information to the identification of the facial expression as negative or positive). For the VRT data, the female positive stimulus face was identified more rapidly than other stimulus faces.

\section{COMMUNICATION FUNCTION}

To achieve a communication function, facial movements have to be rapidly processed and decoded. In their study Kirourac and Dorê (1984) used slides prepared in Ekman (1988) to test the responses of 20 university students. They used six emotions (happiness, surprise, disgust, anger, sadness and fear) and required each of the subjects to press one of six keys corresponding to the emotion displayed on the screen. Their results showed that humans could accurately abstract emotional information from facial stimuli available for extremely short intervals. Included in their procedures were visual masks (visual masking is a technique used in detection experiments that theoretically interferes with the processing of an image or the reduction of the visibility of one stimulus, called the target, by a spatio-temporally overlapping or adjacent second stimulus, called the mask) which quickly followed the target face (Ambada, 2005). The data were compiled and descriptive statistics were generated to test the dependent (subject's response time) versus independent (target affect, distractor affect, and gender of target) variables. Repeated-measures of analysis of variance suggested a statistical difference in judgment associated with the independent variables.

General results for female subjects, with variables, Distractor*Gender, yielded F(1,23) 4.704, p K .05, and with variables Target*Gender, yielded $\mathrm{F}(1,23)=4.043$, $\mathrm{p}<.06$. General results for male subjects, with variables, Target*Distractor*Gender, yielded $F(1,25) 0.369, \mathrm{p}<.06$, began to indicate a pattern wherein the distractor appeared to play a role in the test results. Both genders showed a quicker response time when processing a happy face of the 
same gender. Male response times were faster, overall, than female response times.

Both male and female results showed the fastest times when the target face and the distractor face were both happy. It was not until analysis of the data was being done that the researcher discovered that no measures had been taken for the gender of the subject on an individualized basis. Thus, the reaction times recorded, while measured on an overall average for males and females as separate groups, had not been recorded for the gender of the subject. In addition, the researcher was not convinced that the population size of the study was large enough to adequately allow interpretation on a generalized basis.

The experiment, therefore, was amended to include the gender of each subject as well as the gender of the target and distractor faces as well as to increase the population size. To increase the population size, an additional 50 subjects were chosen who had not taken part in the previous experiment. By disallowing repeat testing, the researcher wanted to avoid an unwanted familiarity with the same facial images. In addition, a third area of study was also included, the percentage of correct responses, allowing a more in-depth understanding of the subjects' recorded responses.

Results of the second experiment indicate that the distractor did, indeed, create a distraction for the subjects. General results for analysis of variance for percent correct with variables Subject Gender*Affect, yielded $\mathrm{F}(1,68)=7.800, \mathrm{p}<.01$. General results of analysis of variance for median reaction time with variables, Affect yielded, $\mathrm{F}(1,68) 13.10, \mathrm{p}<.001$ and with variables Target, yielded $\mathrm{F}(1,68)=8.457, \mathrm{p}<.005$ and with variables Affect*Tg, yielded $F(1,68) 3.3805, p<.01$. The initial design, therefore, should have been more likely to elicit an attentive search when responding to the target stimulus. Results further show that without the distractor, both male and female subjects processed happy male faces most quickly. Happy female faces were second in processing time. Without the distractor, the percentage correct for the affect of the target gender was higher for an angry male target than the other target affects.

Experiments Ia and Ib would seem to further studies conducted by Stanners, Byrd and Gabriel (1985), that there is strong evidence to show that females identify pleasant female facial expressions more quickly than any other target/subject combination. Experiment II, however, conducted without a distractor, presented an unexpected female preference for happy male faces. This result would point more toward Zajonc's theory of an early affective (likedislike) reaction to the image, rather than a feature discrimination process.

There remains a question arising from the results of Experiment II as compared to the results of Experiments Ia and Ib. When the target face is the only focal point, meaning that it is within its own context and there is no distractor, why is the male subject response time significantly faster and why does the female subject now choose the happy male face? Is it possible that without the distractor, the male is now focused on the target more intensely?

Further, is it possible that, without the distractor, the female is now seeking out the friendly, safe face of a male provider?

\section{INFORMATION SYSTEMS}

In relating the results of this study to computer information systems, we will first examine cognitive psychology, or how we gain information, how much and how that information is transformed into knowledge and used to direct our attention and behavior. For the male subject, in all experiments, a happy male face was identified more quickly, regardless of distractor. More importantly, when the distractor was eliminated, the male response time dropped significantly. This would imply that a happy male computer image displayed to a male computer user would stimulate production and efficiency in tasks requiring artificial intelligence computer communications with a male subject user. Visual storage of information stemming from an ongoing "conversation" with a male human image on the screen would serve to keep the image on the screen longer, the added stimulus of auditory sensation would further enhance the storage of the information conveyed by the computer. 
The perception of a familiar, comfortable face would allow the male user to become accustomed to "conversing" with a familiar face, forcing out the contextual cues and allowing concentration on the target, distinguished from competing normal variation. From the results of Experiments La and Lb, the same would hold true for the female subject faced with a happy female computer displayed image in those instances where there is background information being transmitted (distractors). However, the results of Experiment IL indicate that the male user will potentially respond more quickly than a female user, but that both will respond well to a happy male face displayed in a single display environment. The human factors specialist should bear these findings in mind when engineering and designing not only the user interface to be used by the computer system, but the ergonomics of the system as well.

\section{CONCLUSIONS}

Research for this paper suggests that judgment of the system will be based on the affect it produces in the user, be that male or female. In designing human-computer interactive systems, no longer is a simple Graphical User Interface (GUI) sufficient. The designer should engineer a system that is designed ergonomically to use the graphical human face and expression most likely to cause an efficient, pleasant response in the user. Given the design specifications (single point of focus versus background distractor information) and the user community (male or female), the computer-displayed face would vary dependent solely on the purpose and user of the specific application.

Under the conditions of two computer displayed faces (i.e., target and distractor) presented simultaneously to both male and female subjects, it was found that a happy face was processed more efficiently. Under the same conditions, it was found that males processed happy male faces more quickly and females processed happy female faces more quickly. Each subject, when given a choice, identifies with their own particular gender, indicating a gender affect for computer displayed facial images. Under the conditions of a single computer displayed face presented to both male and female subjects, it was found that the male subjects' average response time decreased significantly without the distractor. Given the same experimental circumstances, however, the female subjects' response time remained approximately the same.

Results indicate that female subjects process facial images in parallel (preattentive processing) while male subjects process facial images serially (attentive processing). In the design of interactive visual displays, the human factors specialist needs to utilize a happy male face icon in a single display environment for the best overall reaction time, regardless of the gender of the user. Improved response times equate to increased interactivity and productivity for the end-user community.

\section{AUTHOR INFORMATION}

Ralph T. Reilly earned his Ph.D at Stevens Institute of Technology, Hoboken New Jersey in 1996.Currently he is a professor of Management Information Systems at the University of Hartford in West Hartford, Connecticut and teaches courses in technology for graduate and undergraduate programs. His research interests are in information security, human computer interface, and technology management. His work has been published in numerous refereed journals.

Andrew B. Nyaboga earned his Ph.D at Stevens Institute of Technology, Hoboken New Jersey in 2000.Currently he is an associate professor of Accounting and Law at William Paterson University in Wayne New Jersey and teaches courses in accounting and accounting Information System. His research interests are in technology management, knowledge management and strategic Managemnt. His work has been published in numerous refereed journals.

C. S. Guynes is a Regents Professor of Business Computer Information Systems at the University of North Texas . He received a doctorate in quantitative analysis from Texas Tech University . Dr. Guynes' areas of specialization are client/server computing, end-user computing, data administration, and information resource management. His most recent research efforts have been directed in the areas of client/server computing and data administration. Some of 
the journals in which Dr. Guynes has published include and Communications of the ACM, Information \& Management, The Journal of Information Systems Management, Journal of Accountancy, Journal of Systems Management, The Journal of Database Management, The CPA Journal, The Journal of Computer Information Systems, Information Strategy, Computers and Security, and Computers and Society.

\section{REFERENCES}

1. Ambadar Z, Schooler JW, Cohn JF., (2005). Deciphering the enigmatic face: The importance of facial dynamics in interpreting subtle facial expressions. Psych Sci 16: 403-410

2. Bourne VJ (2005). Lateralised processing of positive facial emotion: sex differences in strength of hemispheric dominance. Neuropsychologia 43: 953-956

3. Buck, R. A., (1979). Measuring Individual Differences in the Nonverbal Communication of Affect: The Slide-Viewing Paradigm. Human Communication Research. 6, 47-57.

4. $\quad$ Buck, R., Miller, R. E., Caul, W. F., (1974). Sex, Personality, and Physiological Variables in the Communication of Affect Via Facial Expression. Journal of Personality and Social Psychology. 30, 5 87596. Johns Hopkins University Press, in press.

5. Burton, L. A., \& Levy, J., (1989). Sex Differences in the Lateralized Processing of Facial Emotion. Brain and Cognition 11, 210-228.

6. Davitz, J. B., (1964). A Review of Research Concerned With Facial and Vocal Expressions of Emotion. in J. R. Davitz (Ed.), The Communication of Emotional Meaning. New York: McGraw-Hill.

7. Ekman, P., \& O'Sullivan, M., (1988). The Role of Context in Interpreting Facial Expression: Comment on Russell and Fehr. Journal of Experimental Psychology: General. 117, 86-88.

8. Fasel, B., Luettin, J., Automatic Facial Expression Analysis: A Survey, PR(36), No. 1, January 2003, pp. 259-275.

9. Hall, J. A., (1984). Nonverbal Sex Differences: Communication Accuracy and Expressive Style. Baltimore, MD: Johns Hopkins University Press.

10. Harrison, D. W., Gorelczenko, P. M. , \& Cook, J., (1990). Sex Differences in the Functional Asymmetry for Facial Affect Perception. Intern. J. Neurosciences. 52, 11-16.

11. Hillger, L. A., Koenig, 0., (1991). Separable Mechanisms in Face Processing: Evidence from Hemispheric Specialization. Journal of Cognitive Neuroscience. 3(1) 42-58.

12. Landis, I., Assol, G., \& Perret, E., (1979). Opposite Cerebral Hemisphere Superiorities for Visual Associative Processing of Emotional Facial Expression and Objects. Nature London, 179739-740.

13. Safer, M. A., \& Leventhal, H., (1977). Ear Differences in Evaluating Emotional Tones of Voice and Verbal Content. Journal of Experimental Psychology: Human Perception and Performance. 3, 75-82.

14. Stanners, B.. F., Byrd, D. M., \& Gabriel, B.., (1985). The Time It Takes To Identify Facial Expressions: Effects Of Age, Gender of Subject, Sex of Sender, and Type Of Expression. Journal of Nonverbal Behavior 9(4), Winter 201-2 11

15. Strauss, E., \& Moscovitch, M., (1981). Perceptual Asymmetries in Processing Facial Expression and Facial Identity. Brain and Language 13: 308-332.

16. Tagiuri, B., (1969). Person Perception. In G. Lindzey \& Aronson (Eds. Handbook of Social Psychology (2nd edition. Vol. 3) Reading, Mass.: Addison-Wellsley.

17. Zajonc, B.. B., (1980). Feeling and thinking: Preferences Need No Inferences. American Psychologist. 35, 151-175.

18. Zeng, Z.H., Pantic, M., Roisman, G.I., Huang, T.S., A Survey of Affect Recognition Methods: Audio, Visual, and Spontaneous Expressions, PAMI(31), No. 1, January 2009, pp. 39-58. 